\title{
Paediatric liver transplantation in Johannesburg revisited: 59 transplants and challenges met
}

\author{
J Loveland, ${ }^{1}$ MB BCh, FCS (SA), Cert Paed Surg; R Britz, ${ }^{1}$ MB BCh, DA (SA), FCS (SA); \\ C Joseph, ${ }^{1}$ MB BCh, DA (SA), FCS (SA), Cert Surg Gastro; A Sparaco, ${ }^{1}$ MB BCh, FCS (SA), Cert Surg Gastro; \\ M Zuckerman, ${ }^{1} \mathrm{MB}$ BCh, FCP Paed (SA); A Langnas, ${ }^{2}$ DO; G Schleicher, ${ }^{3}$ MB BCh, FCP (SA); B Strobele,${ }^{1}$ MB ChB, MMed, FCS (SA); \\ P Moshesh, ${ }^{3}$ MB BCh, FCP Paed (SA), Cert Crit Care (Paeds); J Botha, ${ }^{1}$ MB BCh, FCS (SA)
}

Transplant Division, Wits Donald Gordon Medical Centre, University of the Witwatersrand, Johannesburg, South Africa

${ }^{2}$ Division of Transplantation, Department of Surgery, University of Nebraska Medical Center, Omaha, Nebraska, USA

${ }^{3}$ Intensive Care Unit, Wits Donald Gordon Medical Centre, University of the Witwatersrand, Johannesburg, South Africa

Corresponding author: J Loveland (loveland@wol.co.za)

\begin{abstract}
Background. A paediatric liver transplant programme was started at the Wits Donald Gordon Medical Centre, Johannesburg, South Africa (SA), in November 2005. We reported on the first 29 patients in 2012. Since then we have performed a further 30 transplants in 28 patients, having met the major challenge of donor shortage by introducing a living related donor programme and increasing the use of split liver grafts. Objective. To review the Wits Donald Gordon Medical Centre paediatric liver transplant programme to date. We describe how the programme has evolved and specifically compare the outcomes of the first cohort with the most recent 28 patients.

Methods. Case notes of all paediatric liver transplants performed between 14 November 2005 and 30 June 2014 were retrospectively reviewed. Data were analysed for age and weight at transplantation, indication and type of graft. Morbidity and mortality were documented, specifically biliary and vascular complications. Comparison was made between Era 1 (November 2005 - October 2012) and Era 2 (November 2012 - June 2014).

Results. A total of 59 transplants were performed in 57 patients. Age at transplantation ranged from 9 months to 213 months (mean 82.39 months) and weight ranged from $5 \mathrm{~kg}$ to $62 \mathrm{~kg}$ (mean $21 \mathrm{~kg}$ ). A total of 23 whole livers, 10 reduced-size grafts, 14 split liver grafts and 12 living donor liver transplants (LDLTs) were performed. Eight patients were referred with fulminant hepatic failure (FHF), all in Era 2. Of these, three patients were successfully transplanted. Of the 57 patients, 45 are alive and well with actuarial 1-year patient and graft survival of $85 \%$ and $84 \%$ and 5 -year patient and graft survival of $78 \%$ and $74 \%$, respectively. Sixteen (25.42\%) biliary complications occurred in 15 of our 59 transplants. Seven patients developed significant vascular complications. Comparing Era 1 with Era 2, mean age at transplant decreased from 100.86 months to 64.73 months, mean weight from $25.2 \mathrm{~kg}$ to $16.9 \mathrm{~kg}$, and type of graft utilised changed with a trend away from the use of whole livers and reduced-sized grafts to split livers and segment 2,3 LDLT grafts.

Conclusion. Initially limited by a shortage of donor organs, we aggressively explored optimal utilisation, splitting liver grafts from deceased donors as often as possible and establishing an LDLT programme. This increased access to donor livers allowed us to include patients with FHF and to perform retransplantation in recipients with early graft failure. It remains to offer liver transplantation to the entire paediatric community in SA, in conjunction with the only other established paediatric liver transplant unit, at Red Cross War Memorial Children's Hospital in Cape Town.
\end{abstract}

S Afr Med J 2014;104(11):799-802. DOI:10.7196/SAMJ.8627

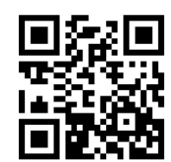

The solid organ transplant unit at the Wits Donald Gordon Medical Centre (WDGMC), Johannesburg, South Africa (SA), performed their first paediatric liver transplant in November 2005. This was a whole liver transplant from a deceased donor. In 2012, the unit published their initial paediatric experience of 29 cases. ${ }^{[1]}$ The major problems identified included the lack of resident expertise in both paediatric intensive care and hepatology, which contributed to the stagnation of the programme for a 2-year period during 2010 and 2011, and a shortage of deceased donor organs. The latter is a worldwide phenomenon and has a direct impact on the ability to transplant not only 'elective' patients on the waiting list with end-stage liver disease, but also those patients requiring urgent transplantation for fulminant hepatic failure (FHF) and retransplant for graft failure. Furthermore, the service excluded transplantation of state patients. Several interventions have culminated in resolving many of these issues. In 2012, the services of a dedicated paediatric hepatologist, a paediatric intensivist and an additional liver transplant surgeon with expertise in living donor liver transplant (LDLT) procedures were secured. The addition of these three specialists, together with a congress dedicated to paediatric hepatobiliary conditions, including liver transplantation, proved to be catalytic in re-establishing and expanding the paediatric liver transplant programme. An additional 30 transplants have been performed since its relaunch in 2012 .

This article serves to review the entire experience to date. We describe how the programme has evolved and how we met the challenges presented.

\section{Methods}

After receiving institutional approval, a retrospective case review including chart analysis was undertaken for all paediatric liver transplants performed at WDGMC between 14 November 2005 and 30 June 2014. Paediatric patients were defined as children $<18$ years of age on the day of transplantation. Data were analysed for patient demographics, including age and weight at transplantation, indication (including patients referred in FHF) and type of graft (whole, reduced, split and LDLT). Biliary and vascular complications, as well as significant medical morbidities and mortality, were also 
documented. Finally, comparison was made between our initial report on Era 1 (November 2005 - October 2012) and the subsequent group of patients in Era 2 (November 2012 - June 2014), specifically documenting age and weight at transplantation, indication (including FHF) and type of graft utilised.

\section{Results}

A total of 59 transplants were performed in 57 patients during the entire study period. Indications for transplant are listed in Table 1. Age at transplantation ranged from 9 months to 213 months (mean 82.4 months) and weight from $5 \mathrm{~kg}$ to $62 \mathrm{~kg}$ (mean $21 \mathrm{~kg}$ ). A total of 23 whole liver transplants, 10 reduced-size grafts, 14 split liver grafts and 12 LDLTs were performed. Eight patients received a combined liver-kidney transplant. ${ }^{[2]}$ Eight patients were referred in FHF, all in Era 2; five patients died of their disease without transplantation, and three patients were successfully transplanted and are alive and well. These included one LDLT and two segments 2,3 split liver grafts. Six state patients have been transplanted in total. Table 2 summarises the differences between Era 1 and Era 2.

\section{Survival}

Of the 57 patients, 45 were alive and well at the time of last follow-up, and actuarial 1-year patient and graft survival were $85 \%$ and $84 \%$ and 5 -year patient and graft survival were $78 \%$ and $74 \%$, respectively (Fig. 1).

Of the 12 deaths, six were in the early postoperative period: one secondary to both hepatic arterial thrombosis (HAT) and portal venous thrombosis (PVT); one secondary to hepatic venous outflow obstruction; one a result of severe acute respiratory distress syndrome 8 days post transplant; and three secondary to severe systemic sepsis and multiorgan failure. The last four patients all died with functioning grafts. The most recent mortality, a 1-year-old child with biliary atresia, received a segments 2,3 split liver graft from a 19-year-old deceased donor and died of primary non-function (PNF) of the graft immediately post transplant.

The remaining six patients died of long-term complications: one of recurrent hepatocellular carcinoma; one of fungal sepsis as a result of persistently high immune suppression managed outside of our unit; two secondary to chronic rejection; one of chronic rejection and multidrug-resistant tuberculosis; and one of fungal sepsis 4 months after retransplant at Red Cross War Memorial Children's Hospital in Cape Town for chronic rejection.

\section{Biliary complications}

Sixteen (25.4\%) biliary complications occurred in 15 of our 59 transplants, including seven anastomotic leaks that required surgical revision and five anastomotic strictures, one of which was revised and subsequently stented percutaneously. The remaining four strictures were treated with percutaneous dilatation and stenting. There were

\section{Table 1. Indications for transplant}

\begin{tabular}{ll}
\hline Indications & $n$ \\
\hline Alagille syndrome & 2 \\
Alpha-1-antitrypsin & 4 \\
Autoimmune & 5 \\
Biliary atresia & 24 \\
Budd-Chiari & 1 \\
Cryptogenic & 1 \\
Cystic fibrosis & 1 \\
Fulminant hepatic failure - Wilson's disease & 1 \\
Fulminant hepatic failure - Hepatitis A & 1 \\
Fulminant hepatic failure - Hepatitis B & 1 \\
Graft failure (HAT) - retransplant & 1 \\
Hepatoblastoma & 2 \\
Hepatocellular carcinoma & 1 \\
HUS & 1 \\
Idiopathic & 1 \\
Maple syrup urine disease & 1 \\
Oxalosis & 4 \\
PFIC & 1 \\
Polycycstic kidney disease and hepatic fibrosis & 3 \\
Veno-occlusive disease & 2 \\
Wilson's disease & 1 \\
Total & 59 \\
HAT = hepatic artery thrombosis; HUS = haemolytic uraemic syndrome; PFIC & primary \\
&
\end{tabular}

\section{Table 2. Comparison by era}

\begin{tabular}{llll}
\hline & Entire series & Era 1 & Era 2 \\
\hline Dates & $2005-2014$ & November 2005 - December 2011 & January 2012 - June 2014 \\
Total transplants & 59 & 29 & 30 \\
Grafts, $n$ (\%) & & & \\
$\quad$ Whole grafts & $23(40)$ & $16(55)$ & $7(23)$ \\
$\quad$ Reduced-size grafts & $10(17)$ & $10(35)$ & 0 \\
$\quad 14(23)$ & $3(10)$ & $11(37)$ \\
$\quad$ Split grafts & $12(20)$ & 0 & $12(40)$ \\
$\quad$ Living donor grafts & $9-214$ & $9-213$ & $9-214$ \\
Age range (months) & 89.4 & 100.9 & 64.7 \\
Mean age (months) & $5-62$ & $5-62$ & $6-45$ \\
Weight range (kg) & 21.0 & 25.21 & 16.9 \\
Mean weight (kg) & 14.1 & 23.0 & 12.8 \\
Median weight (kg) & 6 & 2 & 4 \\
State transplants & 3 & 0 & 3 \\
Fulminant transplants & & &
\end{tabular}




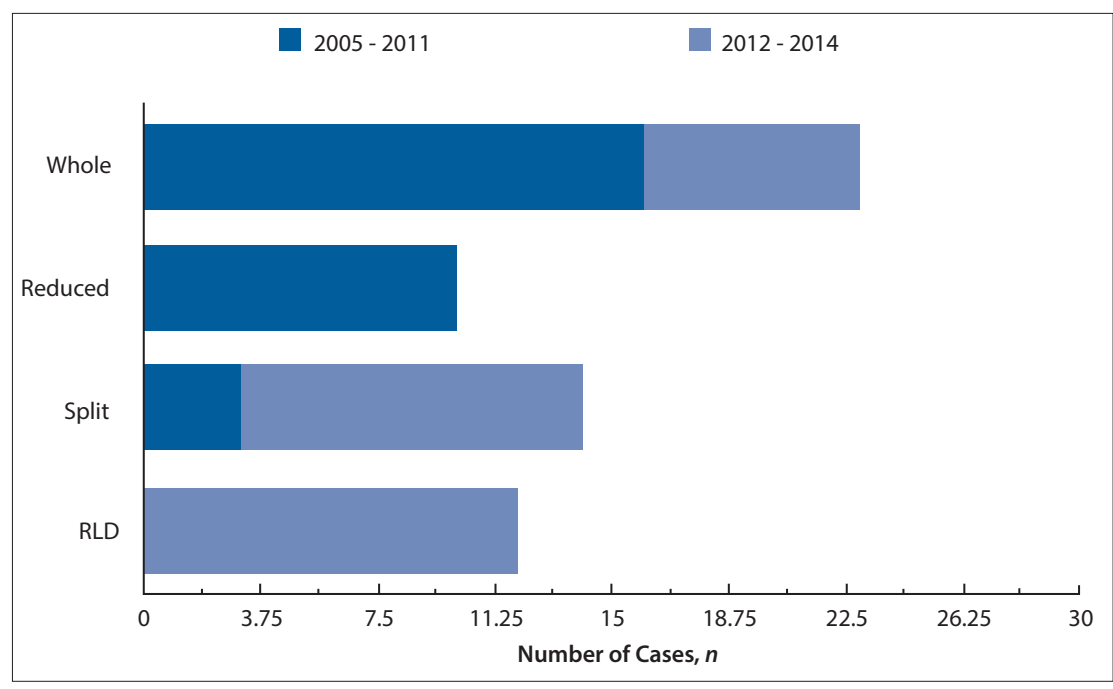

Fig. 1. Graft type by era. Note that in Era 1 predominantly whole livers and reduced-size grafts were used, while in Era 2 there were far fewer whole livers and no reduced-size grafts (which is now largely an obsolete operation), with a significant move towards using more split livers and performing LDLTs. $(R L D=$ related living donor; $L D L T s=$ living donor liver transplants. $)$

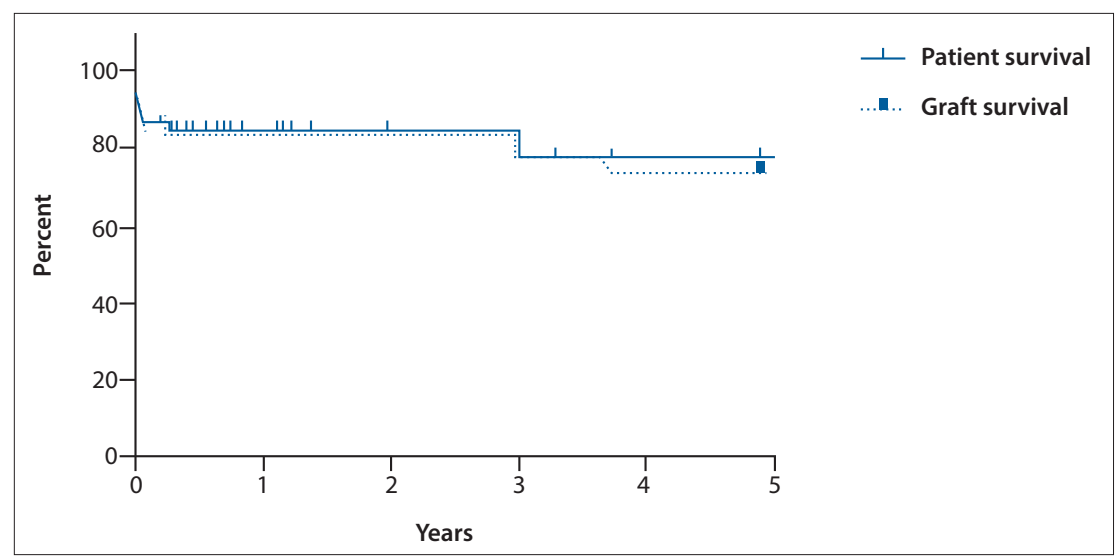

Fig. 2. Kaplan-Meier graph demonstrating actuarial patient and graft survival.

also four cut-surface leaks that required open exploration and drainage, or drainage alone.

\section{Vascular complications}

One patient developed acute hepatic venous outflow obstruction due to torsion of the graft and subsequently died, and a second patient developed both HAT and PVT; both patients died without retransplantation. Two additional patients developed HAT. The first had no sequelae and intervention was unnecessary; he is currently well 7 years post transplant. The other required retransplant for early graft failure. Two patients developed portal vein thrombosis and are currently being managed expectantly, and a third, who developed a symptomatic portal vein stenosis, subsequently required angioplasty and stent. Four patients required reoperation in the immediate postoperative period for bleeding: one from the cut surface, two from the inferior vena cava and one for a diffuse coagulopathy secondary to PNF of the graft.
When comparing Era 1 (29 transplants) and Era 2 (30 transplants), mean age at transplant decreased from 101 months to 65 months, mean weight decreased from $25.2 \mathrm{~kg}$ to $17.0 \mathrm{~kg}$ and the type of graft utilised changed dramatically, with a trend away from the use of whole liver and reduced-sized grafts to split livers and segments 2,3 LDLT grafts (Fig. 2). Four state patients have been transplanted to date in Era 2.

Five of our patients, across both eras, were listed for retransplantation: three for early graft failure, one for chronic rejection and one secondary to HAT. Of the three early graft failures: one patient was relisted for chronic rejection and retransplanted in Cape Town, where he died from chronic rejection and fungal sepsis 4 months after transplant; another patient with HAT (who was retransplanted at WDGMC) died from systemic sepsis 12 days postoperatively with a functioning graft; however, the last patient, a $50 \mathrm{~kg}$ boy with autoimmune hepatitis, was retransplanted 3 days after his initial procedure and subsequently discharged well. Two patients with acute liver failure from Era 1 were not retransplanted as no organs became available; both subsequently died.

\section{Discussion}

Liver transplantation is well established in the treatment of numerous conditions that would otherwise prove fatal; the most common of these is biliary atresia, which accounts for approximately $50 \%$ of paediatric liver transplants performed worldwide. ${ }^{[3]}$ The establishment of a successful liver transplant programme, a quaternary service, is an expensive exercise and requires a multidisciplinary team that works effectively as a cohesive unit from the patient's pretransplant assessment, through the transplant itself, to long-term follow-up. After identifying and addressing the issues in Era 1 that resulted in suspension of paediatric liver transplantation, we relaunched the programme, and in the ensuing 24 months 30 patients were transplanted with overall results comparable with units in the developed world. ${ }^{[4,5]}$

Lack of organ availability remains the limiting factor in transplant programmes worldwide and in $\mathrm{SA}^{[6]}$ and remains the leading cause of waiting list deaths. ${ }^{[7]}$ While not specifically addressed in this paper, our unit is no different. However, the addition of the surgical expertise and experience to perform split liver procedures as well as the LDLT procedures had a significant positive impact on our ability to almost eliminate waiting list mortality (Fig. 2). Such advances not only reduce waiting list mortality, but also allow patients to be transplanted when in better condition. All of the above translated into an increased number of transplants performed, which is evident when comparing the time frames and numbers of our two eras.

Management of FHF in the unit was previously limited by two factors, namely the provision of dedicated paediatric intensive care and, perhaps more importantly, the availability of an organ for transplantation. As noted in the results section, eight patients were referred in FHF in the second era. Although five patients died without an organ becoming available, three of these patients were transplanted, using a split liver deceased donor graft for two and an LDLT graft for the third. This represents a paradigm shift in the management of this category of patient in SA, and as the availability of this treatment option becomes more widespread, we anticipate a significant increase in referrals. As lack of availability of deceased donor organs remains the limiting factor, the LDLT option is a key factor in successful transplantation of these patients. ${ }^{[6,8]}$ 
Should the transplanted graft fail, whether owing to PNF or early graft failure secondary to technical complications (e.g. HAT), urgent retransplant is required. Similar to the situation in FHF, survival of a patient with PNF depends on the availability of an organ for retransplantation. Two of the deaths described in Era 1 would have been prevented had a deceased or LDLT organ been available for transplant. A similar case in Era 2 was successfully retransplanted with a deceased donor liver. The LDLT option does broaden the organ options in the FHF scenario, ${ }^{[0]}$ although it is contentious whether this can be applied in the setting of graft failure after a deceased donor graft.

While LDLT clearly allows access to a far greater pool of organs, it comes with significant ethical issues. ${ }^{[9,10]}$ An obviously life-saving operation for the recipient, the donor operation places an otherwise well individual at risk of morbidity and mortality, documented at 0 - 67\% morbidity (overall average $35 \%$ ) and $0.2-0.4 \%$ mortality ${ }^{[1]]}$ In addition, the decision to donate needs to be made without coercion from other family members; ${ }^{[10]]}$ a meticulous psychosocial evaluation is mandatory prior to anatomical assessment of the potential donor. ${ }^{[10]}$ Having paid close attention to these details, we initiated our LDLT programme in February 2013 with the assistance of a visiting surgeon (Prof. Langnas, Omaha, USA) for our first six cases. We have performed 12 to date and our outcomes compare favourably with those listed in centres in North America ${ }^{[12]}$ From the recipient's perspective, the LDLT option has enormous advantages: the transplant can be performed on an elective basis before the patient's clinical status decompensates; and there is usually an excellent quality of graft with better HLA cross-match, which may lead to better long-term graft and patient survival. ${ }^{\left[{ }^{13]}\right.}$ Added benefits are an increased availability of donor organs and overall reduction in the waiting list mortality for both adults and children.

Biliary complications remain the Achilles heel of liver transplantation and have an increased incidence after both split graft and LDLT when compared with whole liver transplants; this is because of the potential for bile leaks from the cut surface and, in the longer term, an increase in biliary strictures presumably on an ischaemic basis. ${ }^{[1]}$ These are reported to occur in $3-35 \%$ of paediatric liver transplants. ${ }^{[3,4]}$ Our current incidence for the entire series is $24 \%$, falling well within this range. Attention to detail during the LDLT harvest, the back table split of a deceased donor whole liver and reconstruction of the bile duct during the implant procedure, as well as meticulous arterial reconstruction, all contribute to minimising the incidence of this complication. ${ }^{[14]}$

As alluded to in the discussion of our LDLT programme, our unit's current numbers of paediatric transplants per annum compare favourably with other centres in the Western world ${ }^{[12]}$ Looking at the expected number of transplants per population, there is room to grow this significantly. It is well documented that high-volume hospitals are associated with lower morbidity and mortality, and should our trend continue, we have the potential to retain this status for the foreseeable future. ${ }^{[15]}$

Finally, and contentiously, the majority of our patients have been funded by private health insurance, and state patients have not had ready access to liver transplantation in Johannesburg. There are numerous reasons for this, not least of which has been reluctance on the part of the Gauteng Department of Health to recognise our unit and support the referral, care and treatment of these patients in the state sector. It remains our commitment to offer liver transplantation to both fee-paying patients and indigent patients. Six state patients have been transplanted to date, four within the last year. A further six state patients have either been listed for transplant or are in the final stages of their transplant evaluation. In addition, we were recently referred two patients with FHF from the state sector. While no organs became available for these two patients, we were delighted that the precedent had been set and we anticipate successful transplantation of such patients with both chronic and acute disease in the near future. This will include using the living donor option. However, for this to be sustainable, it is imperative that the Department of Health should recognise the need for this treatment modality and provide the necessary support to take this programme forward. It is important to note that selection for transplantation is strictly based on blood group and thereafter severity of disease.

\section{Conclusion}

Our initial paediatric liver transplant programme (Era 1) was a successful and rewarding endeavour. While it provided acceptable outcomes, it also highlighted the logistical issues that limited the unit's growth and development.

After identifying and addressing these factors, our unit has emerged stronger, having more than doubled the number of transplants performed in the more recent cohort (Era 2). In an environment limited by the availability of deceased donor organs, we have aggressively explored optimal utilisation of donor organs, splitting deceased donor organs as often as possible as well as establishing a busy LDLT programme. The consequence of the above has been the ability to assess and transplant patients referred in FHF, as well as to retransplant those rare cases of early graft failure. The unit's results are comparable with well-established international programmes. Having set the precedent, it remains for us to offer liver transplantation to all patients needing transplant who are referred to our unit, encompassing both privately funded and state patients.

Acknowledgements. We would like to acknowledge Antony Beeton, Lliam Brannigan, Mark Eager, Mike Hatchett, Clover-Ann Lee, Rudolph Mononyane and Mike Morford as well as the rest of our anaesthesia team for their unfaltering support. We would also like to acknowledge our dedicated team of transplant co-ordinators and allied medical staff, as well as Ansie Welthagen, Department of Paediatric Surgery, University of the Witwatersrand, for her administrative expertise.

\section{References}

1. Loveland J, Govender T, Botha JR, Britz R. Paediatric liver transplantation in Johannesburg: Initial 29 cases and prospects for the future. S Afr Med J 2012;102(4):233-236

2. Strobele B, Loveland J, Gottlich E, Britz R, Welthagen A, Botha JF. Combined paediatric liver-kidney transplantation: Analysis of our experience and literature review. S Afr Med J 2013;103(12):925-929. [http//dxdoi.org/10.7196/SAMJ7304]

3. Starzl TE, Koep LJ, Schröter GPJ, Halgrimson CG, Porter KA, Weil R. Liver replacement for pediatric 3. Starzl TE, Koep LJ, Schröter GPJ, Halg

patients. Pediatrics 1979;63(6):825-82

B. History of pediatric liver transplantation. Where are we coming from? Where do we stand? Pediatr Transplant 2002;6(5):378-387. [http://dx.doi.org/10.1034/j.1399-3046.2002.01082.x]

LaRosa C, Baluarte HJ, Meyers KE. Outcomes in pediatric solid-organ transplantation. Pediat Transplant 2011;15(2):128-141. [http://dx.doi.org/10.1111/j.1399-3046.2010.01434.x]

6 transplantation at the University of California, Los Angeles. Clin Transpl 1998:255-261.

. Pomfret EA, Sung RS, Allan J, Kinkhabwala M, Melancon JK, Roberts JP. Solving the organ shortage crisis: The 7th annual American Society of Transplant Surgeons' state-of-the-art winter symposium. Am J Transplant 2008;8(4):745-752. [http://dx.doi.org/10.1111/j.1600-6143.2007.02146.x]

8. Slooff MJ. Reduced size liver transplantation, split liver transplantation, and living related liver transplantation in relation to the donor organ shortage. Transpl Int 1995;8(1):65-68. [http://dx.doi.org/10.1007/BF00366715] . Uribe M, González G, Alba A, et al. Living donor liver transplantation in pediatric patients with acute liver failure: Safe and effective alternative. Transplant Proc 2008;40(9):3253-3255. [http://dx.doi. org/10.1016/j.transproceed.2008.03.128]

10. Strong RW, Lynch SV. Ethical issues in living related donor liver transplantation. Transplant Proc 1996;28(4):2366-2369.

11. Nadalin S, Bockhorn M, Malago M, Valentin-Gamazo C, Frilling A, Broelsch CE. Living donor liver transplantation. HPB 2006;8(1):10-21. [http://dx.doi.org/10.1080/13651820500465626]

12. Organ Procurement and Transplantation Network. Paediatric Related Living Donor Transplants 20122013. http://optn.transplant.hrsa.gov (accessed June 2014)

13. Bonanno AP, Saad WA, Ribeiro MA Jr. Pediatric liver transplantation: Comparison between results from deceased and living related transplantation. Transplant Proc 2008;40(3):720-721. [http://dx.doi. org/10.1016/j.transproceed.2008.02.068]

14. Englesbe MJ, Kelly B, Goss J, et al. Reducing pediatric liver transplant complications: A potential roadmap for transplant quality improvement initiatives within North America. Am J Transpl 2012;12(9):2301-2306. [http://dx.doi.org/10.1111/j.1600-6143.2012.04204.x]

15. Tracy ET, Bennett KM, Danco ME, et al. Low volume is associated with worse patient outcomes for pediatric liver transplant centers. J Pediatr Surg 2010;45(1)108-113. [http://dx.doi.org/10.1016/j. jpedsurg.2009.10.018] 